\title{
Equations of State for Mixed Stars
}

\author{
D. P. Menezes \\ Departamento de Física, CFM, Universidade Federal de Santa Catarina, CP. 476, CEP 88.040-900, Florianópolis, SC, Brazil \\ and C. Providência \\ Centro de Física Teórica, Departamento de Física, Universidade de Coimbra, P-3004-516, Coimbra, Portugal
}

Received on 4 September, 2003

\begin{abstract}
We investigate the properties of mixed stars formed by hadronic and quark matter in $\beta$-equilibrium described by appropriate equations of state (EOS) in the framework of relativistic mean-field theory. We use the non-linear Walecka model for the hadron matter and the MIT Bag and the Nambu-Jona-Lasinio models for the quark matter. The calculations were performed for $T=0$ and for finite temperatures in order to describe neutron and proto-neutron stars. The star properties are discussed. Both the Bag model and the NJL model predict a mixed phase in the interior of the star.
\end{abstract}

\section{Introduction and formalism}

Protoneutron stars appear as an outcome of the gravitational colapse of a massive star. During its early evolution a protoneutron star with an entropy per baryon of the order of 1 to 2 contains trapped neutrinos. After 10 to 20 seconds, the star stabilizes at practically zero temperature and no trapped neutrinos are left [1]. The correct calculations of the star properties depend on the appropriate equations of state (EOS) that describe its crust and interior. The crust of the neutron star, where density is low is believed to be adequately described by hadronic matter. Its interior, however, where density is of the order of 10 times nuclear saturation density remains to be properly understood. Whether the central part of the star is composed of quark matter only, of mixed matter or of paired quark matter has been the subject of many works recently.

In this work we investigate the properties of mixed stars formed by hadronic matter at low densities, mixed matter at intermediate densities and quark matter at high densities. We use the non-linear Walecka model for the hadron phase [2] and the MIT Bag [3] and the Nambu-Jona-Lasinio [4] models for the quark matter. We also study the role of the hyperon coupling constants in the appearance of the phase transition to quark matter and the change of strangeness composition with density. Mixed star properties are obtained by solving the appropriate equations for temperatures up to $30 \mathrm{MeV}$.

Most of the formulae used for the lagrangian densities, energies, pressures, partition functions, etc, are not shown in this paper because they are standard equations and can be seen, among others, in reference [5]. For the hadron phase we have used the non-linear Walecka model with the inclusion of hyperons. We have chosen to work with a parametrization which describes the properties of saturating nuclear matter proposed in [6], which we shall call GL force.

In order to fix the meson-hyperon coupling constants we have used several choices discussed in the literature: a) according to [6] we choose the hyperon coupling constants constrained by the binding of the $\Lambda$ hyperon in nuclear matter, hypernuclear levels and neutron star masses $\left(x_{\sigma}=0.7\right.$ and $\left.x_{\omega}=x_{\rho}=0.783\right)$ and assume that the couplings to the $\Sigma$ and $\Xi$ are equal to those of the $\Lambda$ hyperon; b) we take $x_{s B}=x_{v B}=x_{\rho B}=\sqrt{2 / 3}$ as in [7]. This choice is based on quark counting arguments; c) we consider $x_{s B}=x_{v B}=x_{\rho B}=1$ known as universal coupling for comparison [8]. In the results we display, whenever $x_{s B}=x_{v B}=x_{\rho B}$, the unique coupling constant will be called $x_{H}$.

For the NJL model, we consider the set of parameters [9]: $\Lambda=631.4 \mathrm{MeV}, g_{S} \Lambda^{2}=1.824, g_{D} \Lambda^{5}=-9.4, m_{u}=$ $m_{d}=5.6 \mathrm{MeV}$ and $m_{s}=135.6 \mathrm{MeV}$ which where fitted to the following properties: $m_{\pi}=139 \mathrm{MeV}, f_{\pi}=93.0$ $\mathrm{MeV}, m_{K}=495.7 \mathrm{MeV}, f_{K}=98.9 \mathrm{MeV},\langle\bar{u} u\rangle=\langle\bar{d} d\rangle=$ $-(246.7 \mathrm{MeV})^{3}$ and $\langle\bar{s} s\rangle=-(266.9 \mathrm{MeV})^{3}$.

The lagrangian density for the MIT bag model is identical to the one for the leptons, except for the degeneracy factor, which also accounts for the number of quark colors. In the energy density a factor $+B$ and in the pressure a factor $-B$ are inserted. This factor is responsible for the simulation of confinement. For the Bag model, we have taken $\mathrm{B}^{1 / 4}=180$ and $190 \mathrm{MeV}$.

The condition of chemical equilibrium is imposed through the two independent chemical potentials for neutrons $\mu_{n}$ and electrons $\mu_{e}$ and it implies that the chemical potential of baryon $B_{i}$ is

$$
\mu_{B_{i}}=Q_{i}^{B} \mu_{n}-Q_{i}^{e} \mu_{e}
$$

where $Q_{i}^{e}$ and $Q_{i}^{B}$ are, respectively, the electric and baryonic charge of baryon or quark $i$. Charge neutrality implies $\sum_{B_{i}} Q_{i}^{e} \rho_{B_{i}}+\sum_{l} q_{l} \rho_{l}=0$ where $q_{l}$ stands for the electric charges of leptons. In the mixed phase charge neutrality is 
imposed globally,

$$
\chi \rho_{c}^{Q P}+(1-\chi) \rho_{c}^{H P}+\rho_{c}^{l}=0,
$$

where $\rho_{c}^{i P}$ is the charge density of the phase $i, \chi$ is the volume fraction occupied by the quark phase and $\rho_{c}^{l}$ is the electric charge density of leptons. According to the Gibbs conditions for phase coexistence, the baryon chemical potentials, temperatures and pressures have to be identical in both phases, i.e.,

$\mu_{H P}=\mu_{Q P}, T_{H P}=T_{Q P}, P_{H P}\left(\mu_{H P}, T\right)=P_{Q P}\left(\mu_{Q P}, T\right)$,

reflecting the needs of chemical, thermal and mechanical equilibrium, respectively.

As a consequence, the energy density and total baryon density in the mixed phase read:

$$
<\mathcal{E}>=\chi \mathcal{E}^{Q P}+(1-\chi) \mathcal{E}^{H P}+\mathcal{E}^{l}
$$

and

$$
<\rho>=\chi \rho^{Q P}+(1-\chi) \rho^{H P} .
$$

The EOS for the mixed phase are then constructed. Once they are obtained, the properties of the stars can be computed. The equations for the structure of a relativistic spherical and static star composed of a perfect fluid were derived from Einstein's equations by Oppenheimer and Volkoff [10].

\section{Results and discussion}

In Tables 1 and 2 we show the values obtained for the maximum mass of a neutron star as function of the central density for some of the EOS studied in this work and for different temperatures. In Table 1 the GL force and the NJL model were used to derive the full EOS, while in Table 2 the EOS was obtained from the GL force and the MIT Bag model. For all EOS studied, the central energy density $\varepsilon_{0}$ falls inside the mixed phase. In some cases, at finite temperature, it can even occur in the quark phase.

The maximum masses of the stars do not show any regular behavior with temperature, contrary to the steady increase of the maximum allowed mass obtained with the GL model alone as shown in Table 3. The trend seems to be a small decrease at lower temperatures followed by a small increase. Only in one case we have obtained a continuous increase of the mass. Comparing Tables 1 and 2 we conclude that the Bag model allows for smaller maximum masses, of the order $\sim 1.6 \mathrm{M}_{\odot}$, than the NJL model, $\sim 1.9 \mathrm{M}_{\odot}$. It is also seen that the maximum mass does not depend much on the hyperon couplings although in the NJL the same is not true for the onset of the mixed phase. For comparison we show in Table 3 the properties of stars obtained with the GL model (transition to the quark phase not included). While the maximum masses obtained with Bag model are $~ 15 \%$ smaller than with the hadron EOS, the ones obtained within the NJL model are only $~ 3 \%$ smaller.

For all EOS obtained the higher the temperature, the lower the densities for which the hyperons appear. The onset of the mixed phase occurs in most cases studied at higher densities for a higher temperature, the exception being the the NJL model for $x_{H}=\sqrt{2 / 3}$. This can be confirmed in Fig. 1 where the full EOS, obtained respectively with the Bag and the NJL models, for $x_{H}=\sqrt{2 / 3}$, are given for different temperatures. For this choice of hyperon couplings the Bag model predicts a mixed phase at lower densities (energies).

The presence of strangeness in the core and crust of neutron and proto-neutron stars can have important consequences in understanding some of their properties. We have calculated the strangeness content of the different EOS as a function of density. In Figs. 2 we plot the strangeness fraction. When the quark phase is described by the Bag model the strangeness fraction rises steadly and at the onset of the quark phase it has almost reached $1 / 3$ of the baryonic matter. This behavior is independent of the hyperon-meson coupling constants used in this work. The NJL model predicts a different behavior: in the mixed phase the strangeness fraction decreases and increases again for pure quark matter. This behavior is due to fact that for the densities at which the mixed phase occurs the mass of the strange quark is still very high. The overall effect of temperature is to increase the strangeness fraction, except for the mixed phase with the NJL model where the strangeness fraction decreases more strongly for higher temperatures.

TABLE 1. Mixed star properties for the EOS obtained with the GL force and the NJL model.

\begin{tabular}{lccccc}
\hline & $\mathrm{T}(\mathrm{MeV})$ & $M_{\max } / M_{\odot}$ & $\varepsilon_{0}\left(\mathrm{fm}^{-4}\right)$ & $\varepsilon_{\min }\left(\mathrm{fm}^{-4}\right)$ & $\varepsilon_{\max }\left(\mathrm{fm}^{-4}\right)$ \\
\hline$x_{H}=\sqrt{2 / 3}$ & 0 & 1.84 & 6.29 & 4.60 & 7.25 \\
& 10 & 1.83 & 6.34 & 4.58 & 7.14 \\
& 20 & 1.84 & 6.26 & 4.50 & 6.66 \\
& 30 & 1.85 & 5.85 & 4.23 & 5.84 \\
\hline$x_{s}=0.7$ & 0 & 1.90 & 5.01 & 1.30 & 5.21 \\
$x_{\omega}=0.783$ & 10 & 1.89 & 4.98 & 1.31 & 5.13 \\
$x_{\omega}=x_{\rho}$ & 20 & 1.89 & 4.81 & 1.37 & 4.82 \\
& 30 & 1.90 & 4.34 & 1.61 & 4.44 \\
\hline$x_{H}=1$. & 0 & 1.92 & 5.08 & 1.30 & 5.22 \\
& 10. & 1.89 & 5.13 & 1.54 & 5.10 \\
& 20 & 1.88 & 4.92 & 2.20 & 4.83 \\
& 30 & 1.87 & 4.58 & 2.18 & 4.46 \\
\hline
\end{tabular}


TABLE 2. Mixed star properties for the EOS obtained with the GL force and the MIT Bag model.

\begin{tabular}{lccccc}
\hline & $\mathrm{T}(\mathrm{MeV})$ & $M_{\max } / M_{\odot}$ & $\varepsilon_{0}\left(\mathrm{fm}^{-4}\right)$ & $\varepsilon_{\min }\left(\mathrm{fm}^{-4}\right)$ & $\varepsilon_{\max }\left(\mathrm{fm}^{-4}\right)$ \\
\hline $\mathrm{Bag}^{1 / 4}=180 \mathrm{MeV}$ & 0 & 1.40 & 7.38 & 1.17 & 4.62 \\
$x_{H}=\sqrt{2 / 3}$ & 10 & 1.39 & 7.05 & 1.17 & 4.58 \\
& 20 & 1.42 & 4.18 & 1.20 & 4.47 \\
\hline $\mathrm{Bag}^{1 / 4}=190 \mathrm{MeV}$ & 0 & 1.64 & 4.58 & 1.81 & 6.06 \\
$x_{H}=\sqrt{2 / 3}$ & 10 & 1.59 & 4.86 & 1.85 & 6.03 \\
& 20 & 1.67 & 4.18 & 1.90 & 5.93 \\
& 30 & 1.76 & 3.18 & 1.96 & 5.78 \\
\hline$x_{s}=0.7$ & 0 & 1.63 & 4.43 & 1.53 & 6.0 \\
$x_{\omega}=0.783$ & 10 & 1.63 & 4.41 & 1.57 & 5.95 \\
$x_{\omega}=x_{\rho}$ & 20 & 1.63 & 4.10 & 1.62 & 5.86 \\
& 30 & 1.64 & 3.82 & 1.67 & 5.69 \\
\hline$x_{H}=1$ & 0 & 1.64 & 4.49 & 1.63 & 6.01 \\
& 10 & 1.65 & 4.40 & 1.66 & 5.98 \\
& 20 & 1.67 & 4.12 & 1.71 & 5.87 \\
\hline
\end{tabular}

TABLE 3. Compact star properties for the EOS obtained with the GL force

\begin{tabular}{cccc}
\hline & $\mathrm{T}(\mathrm{MeV})$ & $M_{\max } / M_{\odot}$ & $\varepsilon_{0}\left(\mathrm{fm}^{-4}\right)$ \\
\hline$x_{H}=\sqrt{2 / 3}$ & 0 & 1.93 & 6.36 \\
& 10 & 1.93 & 6.34 \\
& 20 & 1.95 & 6.20 \\
& 30 & 1.97 & 5.85 \\
\hline
\end{tabular}

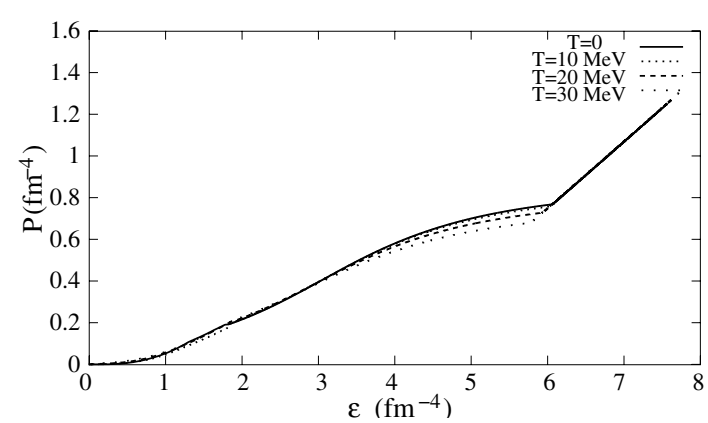

a)

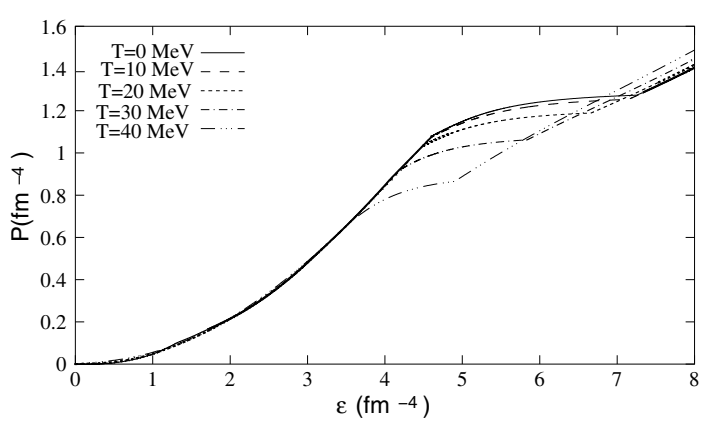

b)

Figure 1. EOS obtained with the GL force plus a) Bag model for $\mathrm{Bag}^{1 / 4}=190 \mathrm{MeV}$; b) NJL model. In both cases $x_{H}=\sqrt{2 / 3}$.
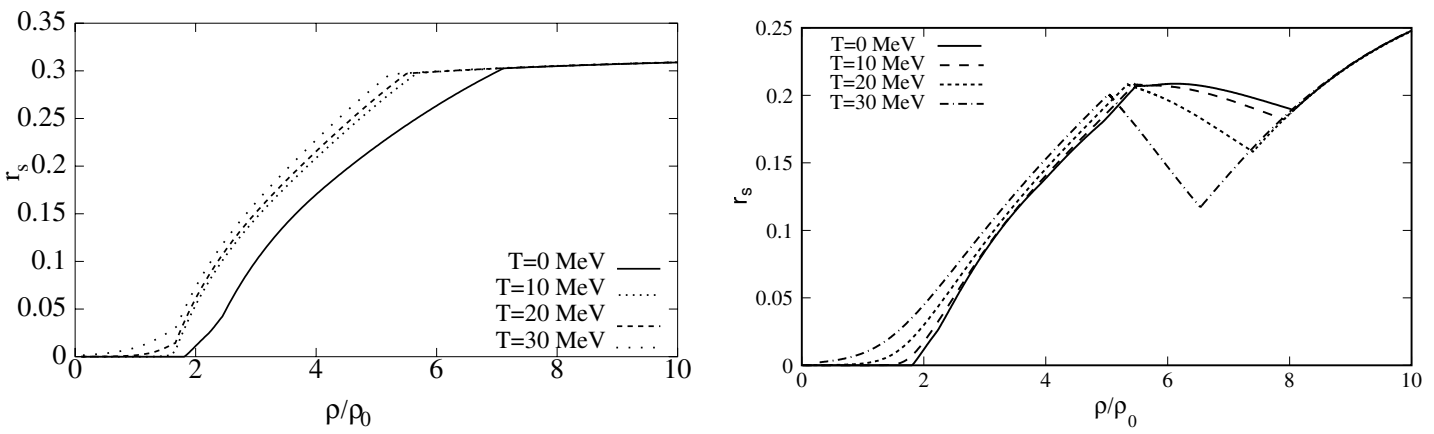

Figure 2. Strangeness fraction $r_{s}$ for the EOS with the a)Bag model for the quark phase with $B^{1 / 4}=190 \mathrm{MeV}$; b) NJL model for the quark phase. In both cases $x_{H}=\sqrt{2 / 3}$. 


\section{Acknowledgments}

This work was partially supported by CNPq (Brazil), CAPES(Brazil)/GRICES (Portugal) under project 003/100 and FEDER/FCT (Portugal) under the project POCTI/35308/FIS/2000.

\section{References}

[1] M. Prakash, I. Bombaci, M. Prakash, P. J. Ellis, J. M. Lattimer, and R. Knorren, Phys. Rep. 280, 1 (1997).

[2] B. D. Serot and J. D. Walecka, Adv. Nucl. Phys. 16, 1 (1995).

[3] A. Chodos, R.L. Jaffe, K. Johnson, C.B. Thorne, and V.F. Weisskopf, Phys. Rev. D9, 3471 (1974).
[4] Y. Nambu and G. Jona-Lasinio, Phys. Rev. 122, 345 (1961); 124, 246 (1961).

[5] D. P. Menezes and C. Providência, Phys. Rev. C (2003) - in press - nucl-th/0308041

[6] N. K. Glendenning, Compact Stars, Springer-Verlag, NewYork, 2000.

[7] S. A. Moszkowski, Phys. Rev. D9, 1613 (1974).

[8] N. K. Glendenning, Astrophys. J. 293, 470 (1985).

[9] C. Ruivo, C. Sousa, and C. Providência, Nucl. Phys. A651, 59 (1999).

[10] R. C. Tolman, Phys. Rev. 55, 364 (1939); J. R. Oppenheimer and G. M. Volkoff, Phys. Rev. 55, 374 (1939). 